\title{
Resolving sill pillar stress behaviour associated with blasts and rockbursts
}

\author{
LT Smith-Boughner ESG Solutions, Canada
}

TI Urbancic ESG Solutions, Canada

AM Baig ESG Solutions, Canada

\begin{abstract}
In this paper, we describe the seismicity associated with the excavation of a stope inside a sill pillar in a North American hard rock mine. By taking a continuum approach to describing the microseismicity, we reconstruct the deformation state of the rock mass during production and production. Microseismic events are generated through dynamic changes to the stress in the rock mass. While an individual microseismic event corresponds to slip on a discrete surface, the combined deformation of a group of events results in a collective behaviour. The combined deformation is quantified using a dynamic parameter representation and allows us to gain additional insight into the behaviour of the rock mass through incorporation of stress and energy release, and spatio-temporal relationships of observed seismicity. It provides a quantitative framework for relating changes in the intensity, concentration and rate of seismicity to changes in the rock mass. In this paper, we show that the bulk of an elastic deformation, for the most part, follows spatially around the major blast locations. Using these tools and seismic moment tensor data for these events, we track changes in the strength of the rock mass in the hours and days following the blasting of a stope. The stresses in the stope are relieved through the excavation and shed to the surrounding rock mass. The timing and mechanism for this stress transfer varies near faults or close to other structural boundaries. Depending on the nature of the interface, the change in the collective behaviour could be a change in the event rate, inter-event distance or energy release of the observed seismicity. Based on our observations, we suggest that the application of dynamic parameters to characterise the seismicity associated with mine design can be used to better characterise the stress state, fracture state and deformation state/evolution of a rock mass.
\end{abstract}

Keywords: microseismicity, rock mass characterisation, stress

\section{Introduction}

Seismic monitoring and analysis is a useful tool for understanding rock properties and stress loading within a mine. Particularly in high stress environments, with the correct instruments seismic analysis can tell you not only where the event was, but the amount of deformation and the radius of the source. By utilising a three-dimensional distribution of three-component geophones, a robust determination of fracture orientation and failure mechanism can be estimated by moment tensor inversion techniques. Armed with this information, we can assess which faults are moving and, by using full six-component moment tensors, if cracks are opening, closing or sliding.

Of importance to mining operations is a better understanding of the stress state of the rock mass. Is the rock critically stressed? What can be done to de-stress the rock mass? To answer these questions, we must understand how close to critically stressed the rock is, the degree of fracturing within the rock, and how the rock will deform under load. By utilising recorded seismicity as a proxy for describing how the rock deforms, we can build an understanding of the stress conditions within a rock, and by characterising the collective behaviour, we can further our understanding of the deformation process being observed (Ben-Zion 2008).

In quantifying the collective behaviour of the seismicity, we can begin to understand the aggregate conditions of the rock mass under examination. For example, some areas experience a lower rate of seismicity but larger 
event magnitudes. Quantifying and understanding differences in the size, spatial concentration and event rate allow us to highlight different stress, fracture and deformation states within the rock mass. By considering the characteristics of clusters of seismicity in a spatiotemporal context, we can estimate the strength of rock mass, how it deforms under loading and stress field responsible for the observed seismicity.

Dynamic parameter characterisations allow differences in the stress, fracture and deformation state within a rock mass to be quantified and tracked. The dynamic parameter characterisation can be applied in real time to image the rock mass during production activities or during periods of unusual seismic activity.

In this paper, we review the seismic concepts of seismic efficiency and introduce the dynamic parameter characterisation. Seismic moment tensors are estimated using the methods described by Trifu et al. (2000). These methods are applied to a case study from a hard rock mine in North America. We utilise the findings to identify variations in the energy budget, types of failures and spatio-temporal pattern of the seismicity during the production and blasting of a stope inside the sill pillar.

\section{Stress versus strain}

At different places on a stress strain rate curve, an increment of additional force results in different behaviour, as shown in Figure 1. Adding stress to a rock mass could result in strain-hardening behaviour, e.g. additional compressive stress adds more energy to the rock by compressing the rock elastically, which resists further deformation (see Hobbs et al. 1990 for a full discussion). Alternately, additional stress could cause the rock to fail. Rather than storing the energy, the rock matrix breaks down. This deformation is unrecoverable and, therefore, plastic. For some materials and rock conditions, additional stress can result in slip-weakening behaviour. As the rock begins sliding, friction is reduced and it is easier to continue sliding (elastic-perfectly-plastic).

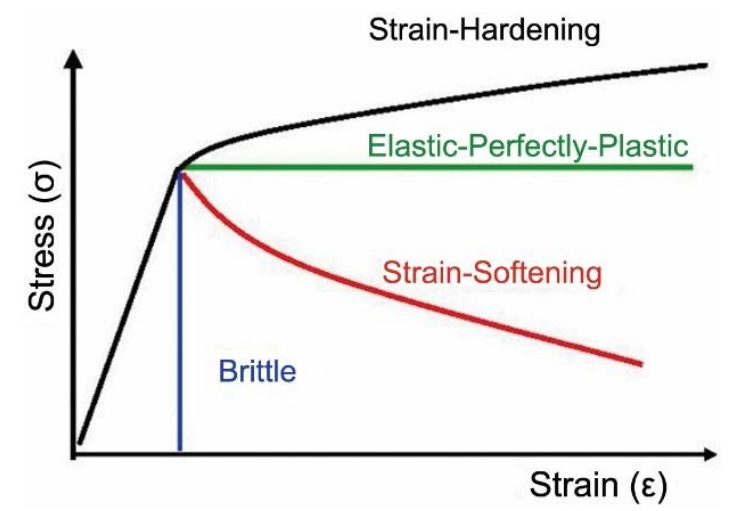

Figure 1 Idealised stress-strain curve showing different end-member behaviour: strain-hardening, elastic-plastic deformation, and strain-softening

Using the waveforms of the compressive and shear waves generated by the rupture process, the dynamics of the rupture process can be examined.

\section{Seismic energy budget}

Seismicity is often quantified in terms of seismic moment and source radius. From recordings of the compressional and shear waves generated by the deformation of the rock mass, the size of the area undergoing deformation and the total deformation (the seismic moment) is estimated. The apparent stress at the time of rupture, $\sigma_{a}$ and the stress released as a result of the deformation, the static stress drop, $\Delta \sigma_{d}$ provide insight into the stress conditions before and after failure.

The radiated energy reflects only a part of the energy involved in the deformation process. As the fault slides, energy is lost due to frictional heating and in extending or generating fractures in the rock mass. The seismic (or Savage-Wood) efficiency, $\eta_{e}$ in Equation (1), provides insight into the energy budget of the observed 
deformation process (Savage \& Wood 1971). It is the ratio of the apparent stress, $\sigma_{a}$, to the static stress drop, $\Delta \sigma_{d}$, and estimates the relative amount of energy radiated by the seismicity and the amount of energy used up the other processes previously outlined (described further in McGarr 1999).

$$
\eta_{e}=\frac{\sigma_{a}}{\Delta \sigma_{d}}
$$

Relative variations in seismic efficiency relate to the ease in generating co-seismic deformation during the rupture process, for example if $\eta_{\mathrm{e}}$ is low, more energy is used for deformation-related processes than for events with higher efficiencies (more efficient at radiating seismic energy). Variations in the energy budget can indicate regions where more energy is used by deformation processes and could indicate slip weakening behaviour.

\section{$4 \quad$ Characterisation using dynamic parameters}

Considering a cluster of events, when deformation occurs on one fault, energy is used for frictional heating and generation of new fracture surface area. The remaining energy involved in the deformation is radiated in the form of seismic compressional and shear waves. When seismicity is confined into a small spatial area, the interaction between radiated waves and local changes in the stress field, due to deformation, influence the dynamics of subsequent ruptures. If events are occurring rapidly, more seismic energy is radiated and changes in the stress of the deformed area can influence the rupture dynamics of subsequent ruptures.

To quantify these interactions, we use a nearest neighbour clustering approach to characterise variations in the timing, spacing and the energy budget of the seismicity. For each cluster of events, several different dynamic parameterisations of the seismicity are compared. This approach seeks to estimate the relative influence of different variations in the seismicity. The diffusion index (DI), estimates the size and rate of the seismicity:

$$
D I=\frac{\hat{x}^{2}}{\hat{t}}
$$

where:

$\hat{X}^{2}$ is the square of the average of the distance between all events within the cluster.

$t$ is the average of the time between all events (from Mendecki 1994).

Usually, a high DI, as shown in Figure 2(a), means that the events are far apart spatially. This spatial separation indicates that the seismically active area is very large, relative to the rate of event occurrence. A low DI indicates that the seismicity is occurring in a compact area, relative to the event rate. The DI provides insight into the fracture state of the rock mass and the spatial concentration of the deforming fractures.

To understand how readily the rock mass deforms, we use the Plasticity Index (PI). This quantity estimates how easily a given volume of rock, $\Delta \mathrm{V}$, deforms within a given period of time, $\Delta \mathrm{t}$, as:

$$
\operatorname{PI}(\Delta V, \Delta t)=\frac{\mu}{\Delta V \eta_{c}}
$$

where:

$\mu$ is the dynamic shear modulus.

$\eta_{c}$ is the cumulative seismic efficiency of the cluster.

A high $\mathrm{PI}$, as shown in Figure 2(b), suggests that, for the volume and timespan under observation, the rock mass deforms easily. A low PI suggests that the rock is more difficult to deform and does so more slowly. 
To understand the stress state within the rock mass, we estimate a Stress Index (SI), the ratio of the elastic to viscous forces responsible for generating the observed seismicity. The Stress Index is defined as:

$$
\mathrm{SI}(\Delta V, \Delta t)=\frac{c}{D I} \eta_{c}
$$

where:

$\eta_{c}$ is the cumulative seismic efficiency of the cluster.

$\mathrm{c}$ is a scaling term to account for the material properties and the time and volume span under consideration.

SI weights the energy budget of the observed seismicity against the spatial concentration of the seismicity within a specific volume and timespan. This helps assess the complexity of the underlying stress field that generated the observed seismicity. In Figure 2(c), we show a depiction of a low SI, where seismicity occurs close together in space and time. Local stress changes from nearby seismic activity results in a rapidly evolving fracture process. These are suggestive of a rapidly evolving and complex stress field. When the $\mathrm{SI}$ is high, the seismicity is wide-spread and evolves slowly, suggesting that the evolution of the stress field is far more stable.

a) DI

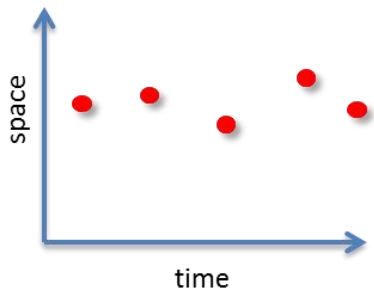

Low DI: Events occur close together in space, with large inter-event times.

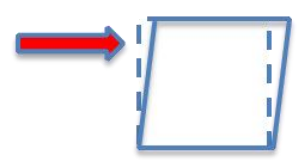

b) PI

c) SI
Low PI: Little deformation in the rockmass when a large driving force is applied.

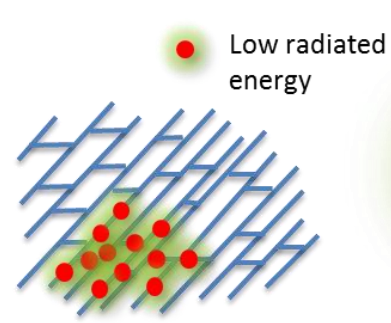

Low SI: Events characteristic of co mplex deformation processes:

- localised in time and space

- low radiated energy.

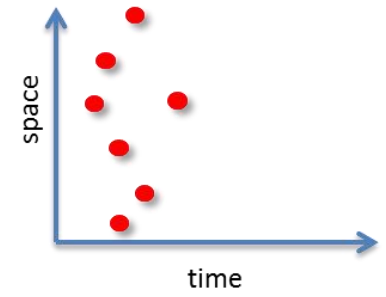

High DI: Events jump around in space, over short inter-event times but large inter-event distances.

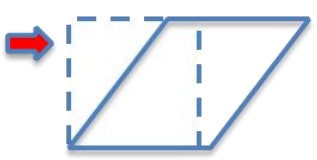

High PI: Rockmass is easily deformed with respect to a small driving force.

High radiated energy

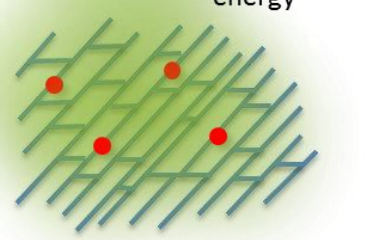

High SI: Events characteristic of stress -triggered seismicity on optimally oriented faults:

- large inter-event times and distances

- high radiated energy.

Figure 2 (a) Diffusion Index (DI); (b) Plasticity Index (PI); and (c) Stress Index (SI)

The dynamic parameters previously described, DI, PI and SI, consider different aspects of the observed seismicity within a region. These concepts are similar to those from Kostrov (1974) and Mendecki (1994), but have been adapted to incorporate clustering approaches to characterise the spatial and temporal variations in the observed seismicity. 
To examine the influence of stress, deformation and fracturing, we plot the three parameters using a ternary diagram, shown in Figure 3, with the significance of each end-member. The distance from the centre of the figure indicates the relative influence of each dynamic parameter on the overall rupture process.
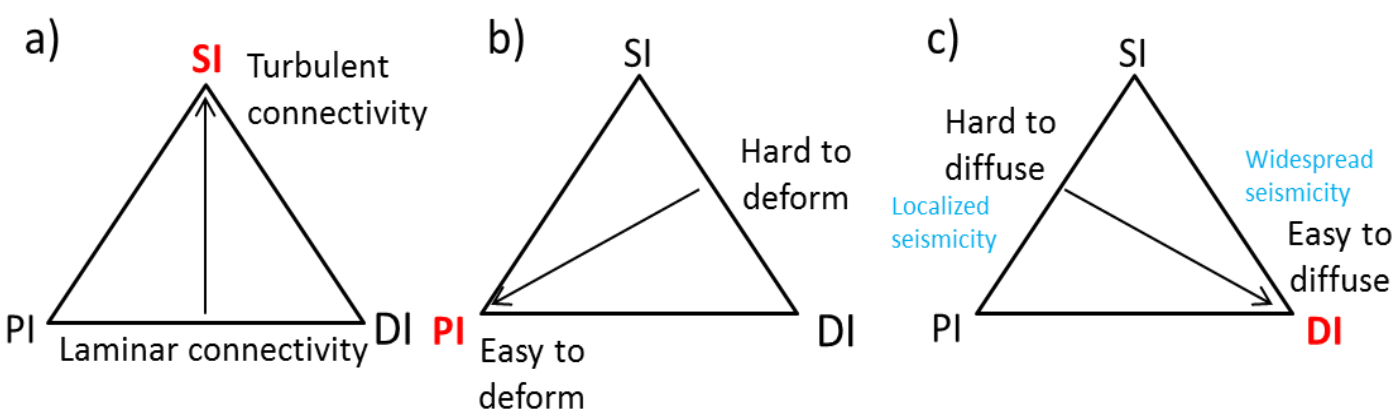

Figure 3 Ternary representation of SI, PI and DI to show the inter-relationships between connectivity, deformation and diffusion

\section{$5 \quad$ Seismic moment tensors}

The evaluation of seismic moment tensors allows for an estimate of the set of forces coupled which describes the observed deformation. Often, most of the observed deformation is the result of shear-slip along a fault. This mechanism can be represented using a double-couple source. In more complicated environments, the volume of the deformed area changes as well. These changes can be represented using a general seismic moment tensor, which considers components of isotropic volumetric change - implosion or explosion, and a volume distortion - modelled using a compensated-linear-vector dipole (CLVD).

For a general seismic moment tensor, the mechanism of failure can be represented visually using a Hudson plot (Hudson et al. 1989). This plot, shown in Figure 4, is used to represent the general seismic moment tensor in terms of the different end-members of failure. Isotropic-volumetric change, either explosion or implosion, is represented as the vertical distance from the origin. A pure shear-slip mechanism, from a double-couple source, would appear at the origin. Many seismic events represent the opening or closing of a shear-tensile crack. Failures of this type would appear in the top left corner of the Hudson plot (shown as (1) in Figure 4) for crack openings, where volume is increased.

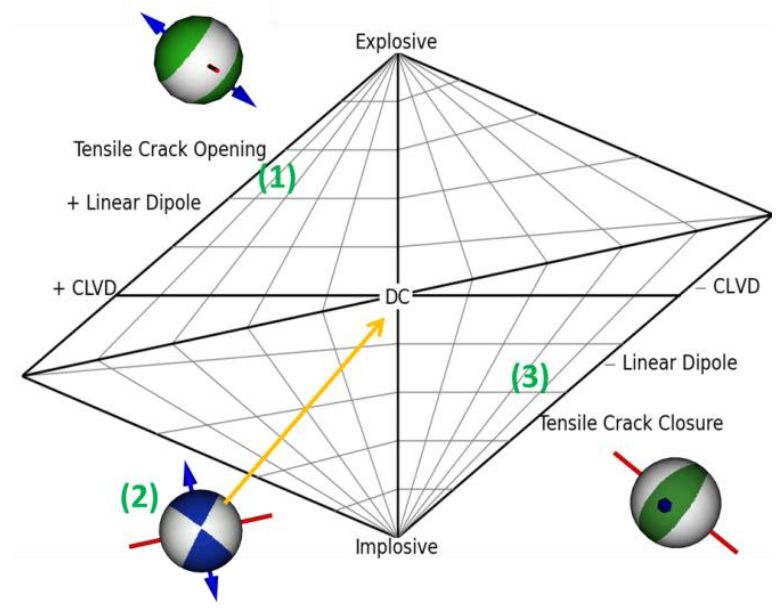

Figure 4 Source-type plot (Hudson et al. 1989). The general seismic moment tensor can be decomposed into different types of failure. The volumetric component is represented by the vertical distance from the origin. The volume-preserving components of motion-shear slip and CLVD (compensated-linear-vector-dipole) are represented as the distance along the horizontal axis. 'Beach ball' representations for: (1) shear-tensile crack openings; (2) shear events; and (3) shear-tensile crack closures are shown 
The shear-tensile crack model, shown in Figure 5, from Vavrčyuk (2001), characterises the geometry of the failure. Slip, [u] occurs at an angle, $\alpha$ to the failure surface (marked as $\Sigma$ ). The tensile angle, $\alpha$ is positive for when the crack is opening and negative if the crack is closing.

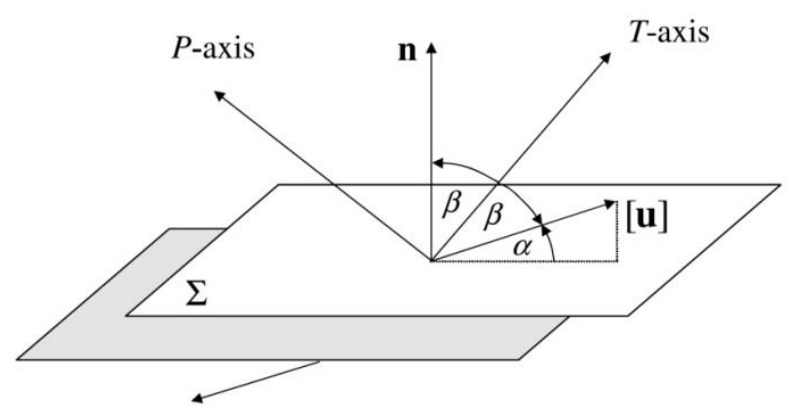

Figure 5 Sketch of the motion of the shear-tensile crack. Figure from Vavrčyuk (2001). The direction of compressive (extensive) strain is denoted as the P-axis (T-axis). For a shear-tensile crack, the fault surface, labelled as $\Sigma$, slip at an angle, $\alpha$ (referred to as the tensile angle) to the fault surface. If the crack is opening, the tensile angle is positive and the volume of the focal sphere increases

\section{Case study - deep hard rock mine}

Using data recorded on 15 triaxial geophones in a hard rock mine in Canada (shown in part in Figure 6), we studied the production of a stope. The resulting seismicity is analysed using seismic moment tensors and the dynamic parameters, $\mathrm{DI}, \mathrm{PI}$ and $\mathrm{SI}$, to characterise the observed seismicity and changes in deformation, stress and fracturing near the stope.

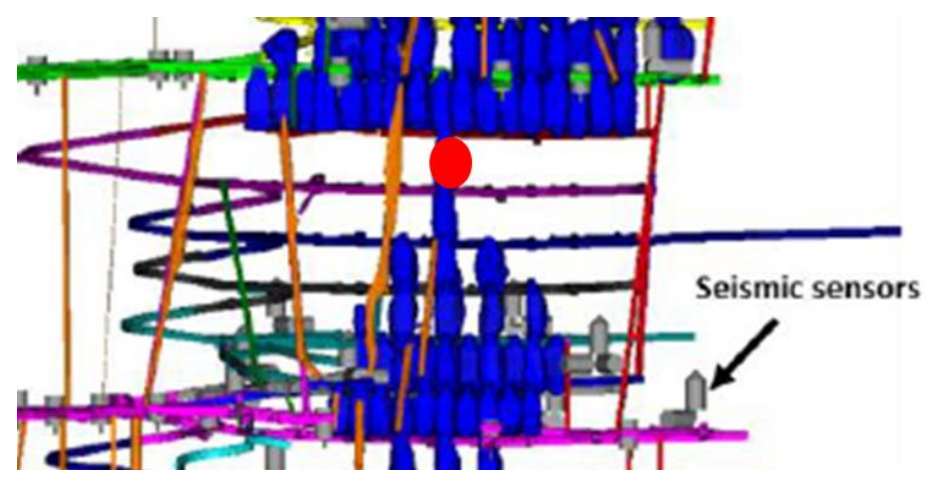

Figure 6 A close-up of the layout of seismic network around the area of interest is shown. The stope under-study is shown in red. Triaxial geophones and uniaxial accelerometers for this part of the network are shown in grey

At this point in the mine sequence, the rock above and below has been extensively mined out. Two stopes to the North have already been mined out from the sill pillar several months before. The final stope in the panel was developed in two parts, an initial blast at the toe of the stope, followed by a final production blast 18 days later (Figure 7). After each blast, there was a burst of seismicity, with far more events occurring to the west of the stope (Figure 8). Between the blasts, a large rockburst, with $\mathrm{M}_{\mathrm{w}} 1.5$ (Moment magnitude, after Kanamori 1983) occurred in sill pillar near the stope, shown as a yellow star in Figure 8. 


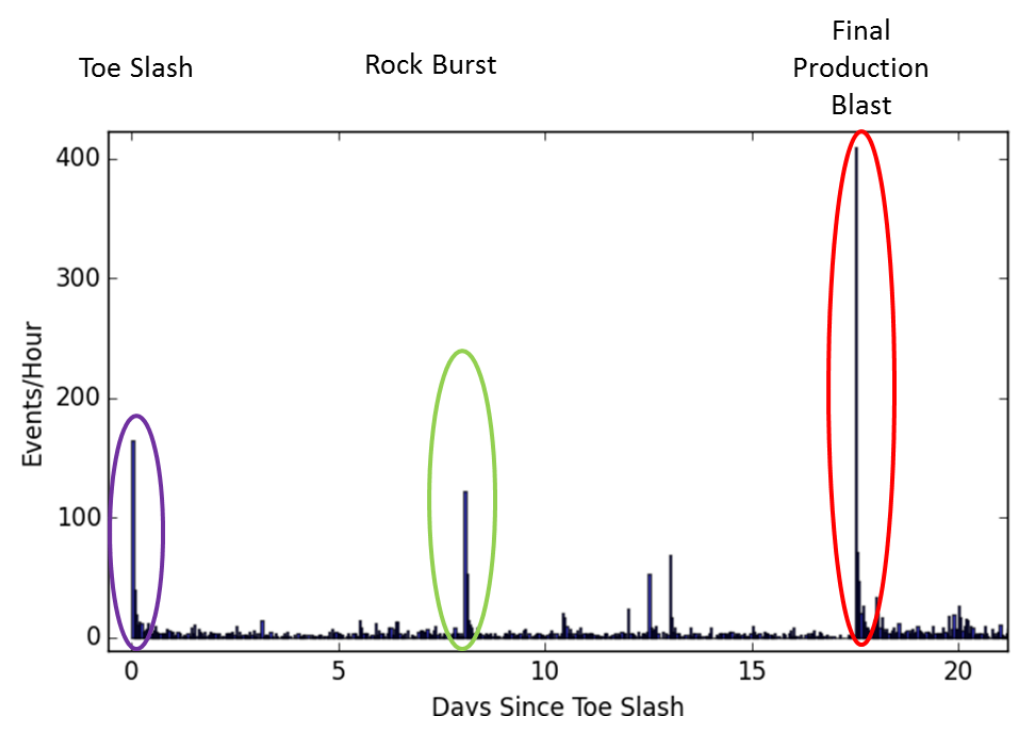

Figure 7 Rate of seismic events per hour, shown following the initial toe slash blast of the stope. A rockburst occurred seven days later in the pillar above the stope. When the final production blast was carried out, there was a cluster of seismicity in the hour following the blast

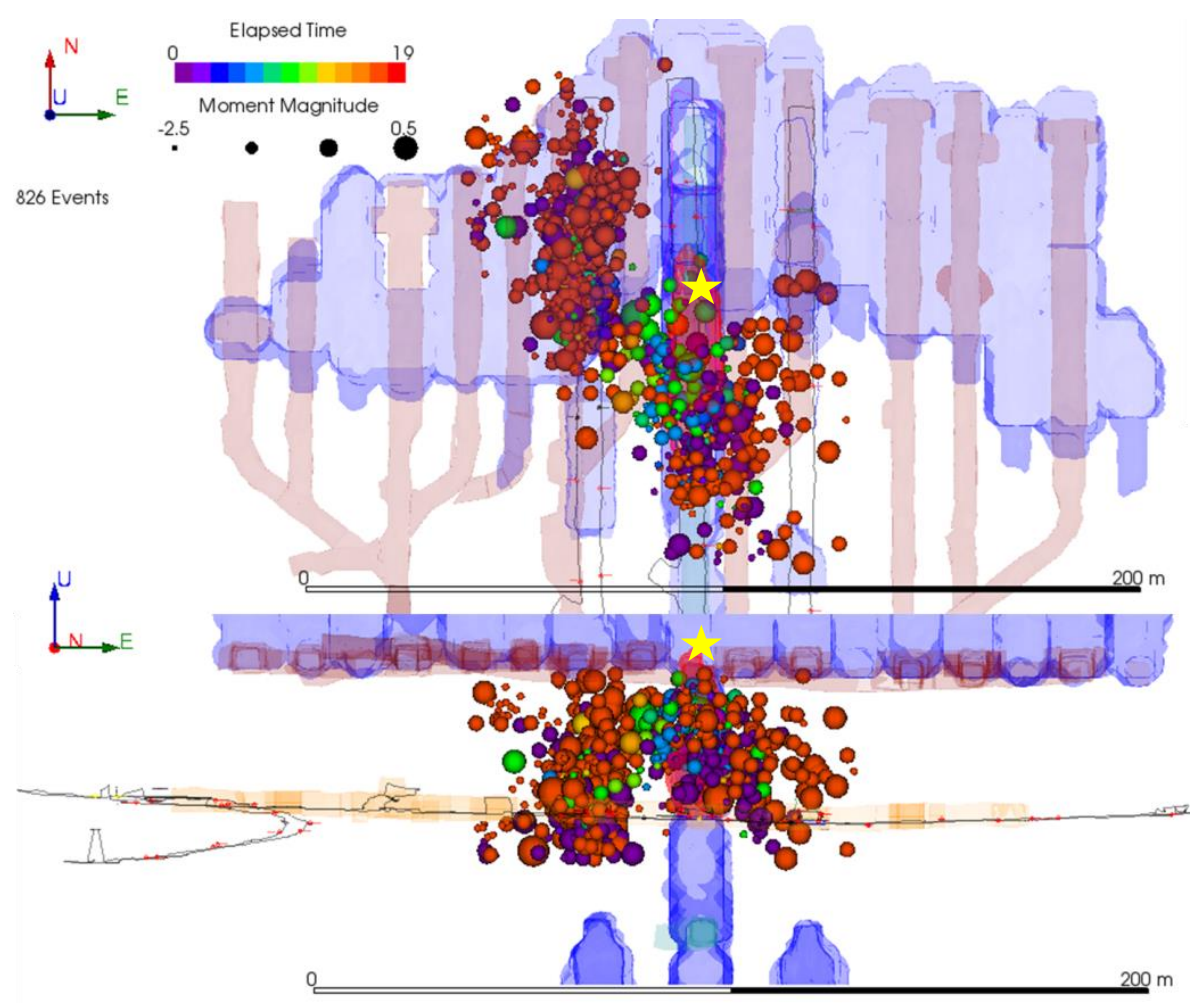

Figure 8 All case study events: coloured by the elapsed time (in days) since the first production blast (stope shown in red). The blue regions in the view indicate previously mined and back-filled stopes. The location of the large rockburst is shown in yellow

\subsection{Analysis of seismicity following final production blast}

The seismicity following the final production blast is shown in Figure 9. The events on the west side of the stope have low seismic efficiency values, implying proportionately more of the energy of the rupture was used in the deformation processes than was radiated. To model the mechanism of failure, general seismic moment tensors were estimated for the events shown in Figure 9. Most of these failures represent shear-tensile failures. To the west of the stope, shown in the left panel of Figure 10, the failures are 
dominated by shear-tensile crack opening failures, with few shear-tensile crack closures. Most of the opening events occur within the first 10 minutes after the blast and show significant increases in volume. After this initial relaxation period, seismicity to the west of the stope is due to the closure and compaction of tensile cracks. The rapid transition from a stress state dominated by extensional forces to one driven by compressional forces is limited to the region west of the stope. Seismicity to the east of the stope, shown in the right panel of Figure 10, is related to the compaction and closure of shear-tensile cracks.

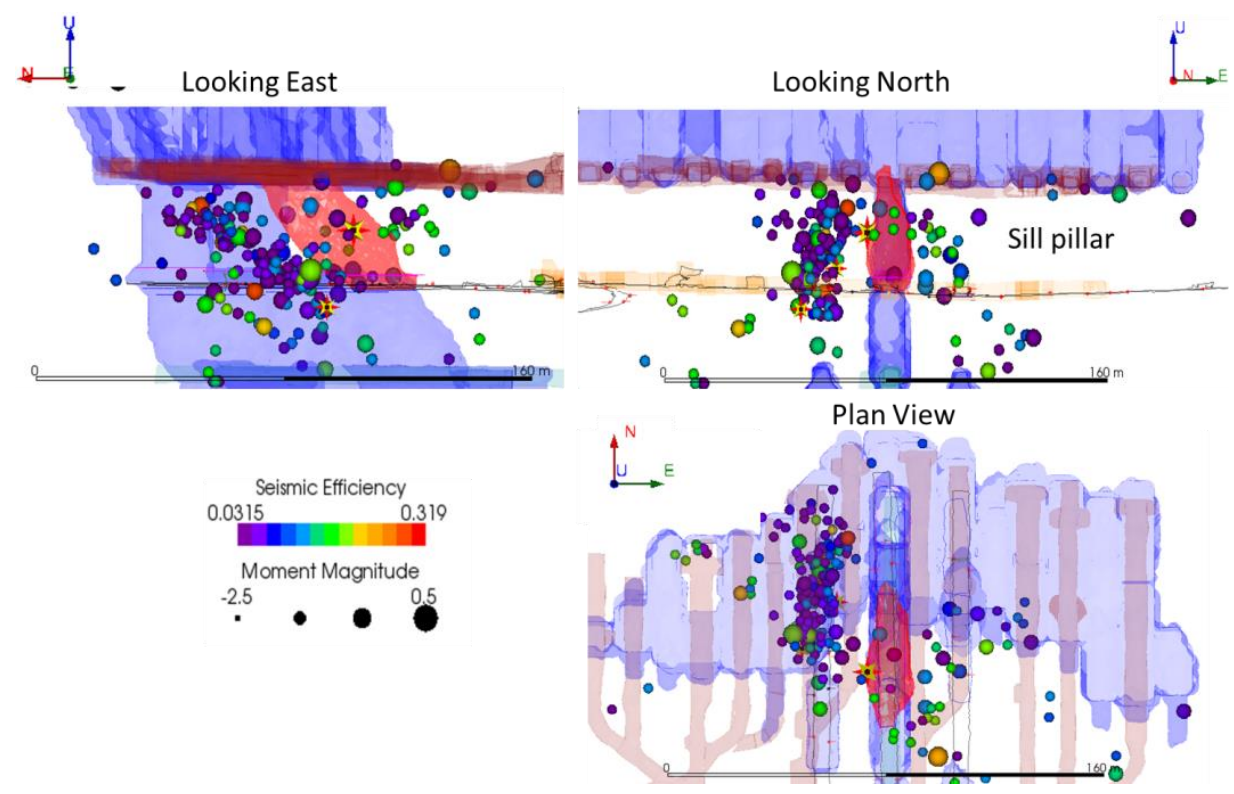

Figure 9 Seismic Moment Tensor Inversions of case study events: after final production blast inside stope (shown in red). Seismic moment tensors were estimated for all the events shown

a)

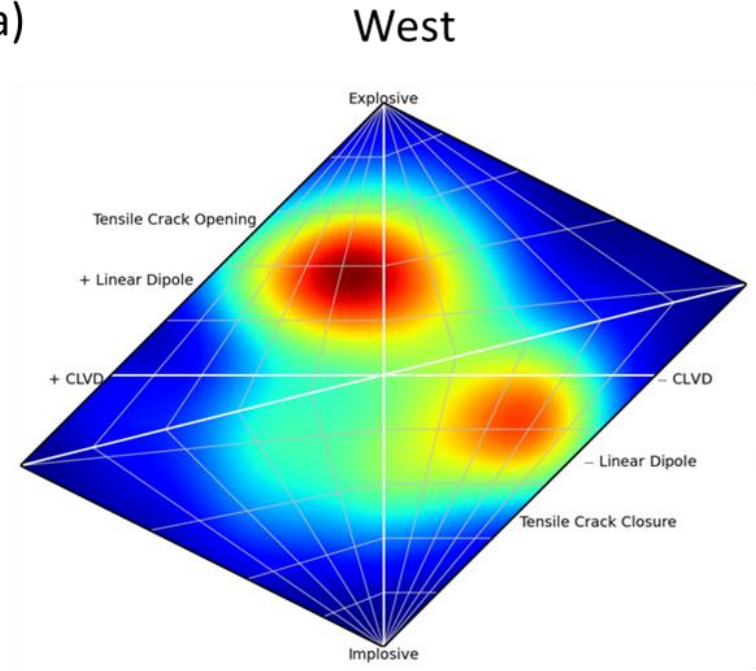

b) East

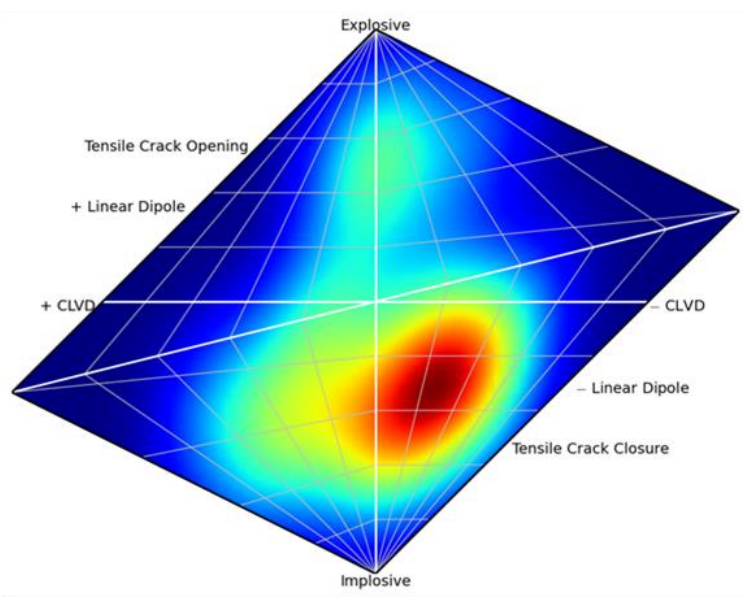

Figure 10 Source-type (Hudson 1989) plot showing density of the observed source-types; (a) most of these events correspond to the opening of a shear-tensile crack, with some events being caused by the closure of shear-tensile cracks; (b) mechanisms to the east correspond to the closure of a shear-tensile crack

\subsection{Dynamic parameter characterisation post final production blast}

Utilising the dynamic parameters described in Section 4, we cluster the events using a rolling nearest-neighbour grouping of events and estimate the $\mathrm{DI}, \mathrm{PI}$ and $\mathrm{SI}$ for all events observed within this region in the 24 hours following the blast. To quantify the impact of the observed seismicity on the area around the stope, we plot these in Figure 11 using a ternary diagram. The same clusters are plotted twice in Figure 10, 
on the left they are coloured by the mean tensile angle (shown as $\alpha$ in Figure 5, from Vavrčyuk 2001) of the cluster and on the right they are coloured by easting coordinate of the centroid of the cluster. Viewed in this way, we are considering the impact of all nearby seismicity during the day. Many of the clusters with shear-tensile closures have very high $\mathrm{PI}$ and low DI, indicating that they are very easy to deform and occur in a spatially compact region. Regions dominated by shear-tensile openings tend to have higher DI and lower $\mathrm{PI}$, indicating that it is more difficult for the rock mass to deform and that it is far less fractured. Clusters to the east of the stope (plotted in yellow colours on the right panel of Figure 11) correspond to a mixture of shear-tensile openings and closures. Clusters that are centred on the stope have a higher DI, indicating that the seismicity here is very spatially dispersed.

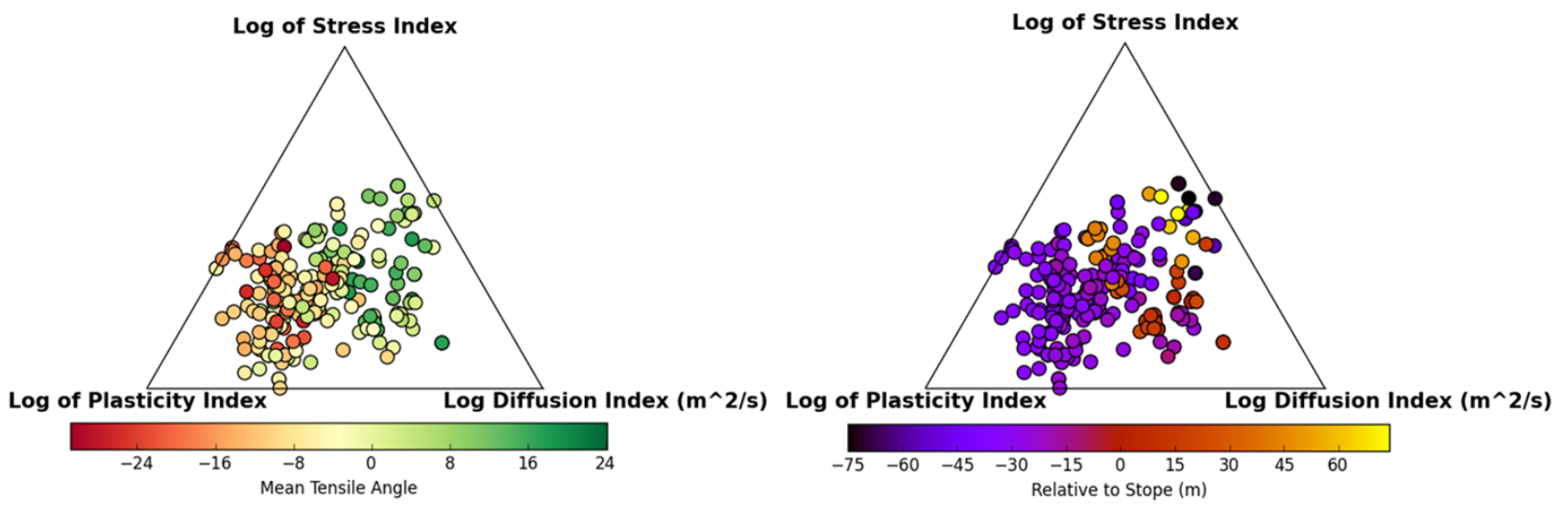

Figure 11 Ternary plot of dynamic parameterisation of the events shown in Figure 9; left: events are coloured by the mean tensile angle of the cluster; right: events are coloured by the easting coordinate of the cluster, relative to the stope

\subsection{Dynamic parameter characterisation for stope production}

Dynamic parameters were estimated for all events that occurred between the two blasts, as shown in Figure 9 . Events were clustered using a nearest-neighbour approach and a one hour moving window. After the toe slash, the first events have very high $\mathrm{PI}$, indicating easily deformed rock, and low $\mathrm{SI}$, indicating that the stress field is evolving rapidly, shown with diamonds on the ternary diagram in Figure 12. One hour later, the DI has increased and events are occurring further from each other. The stress field is evolving more slowly, inferred from the lower SI. At the end of the day, the clusters have very low PI indicating little deformation is occurring.

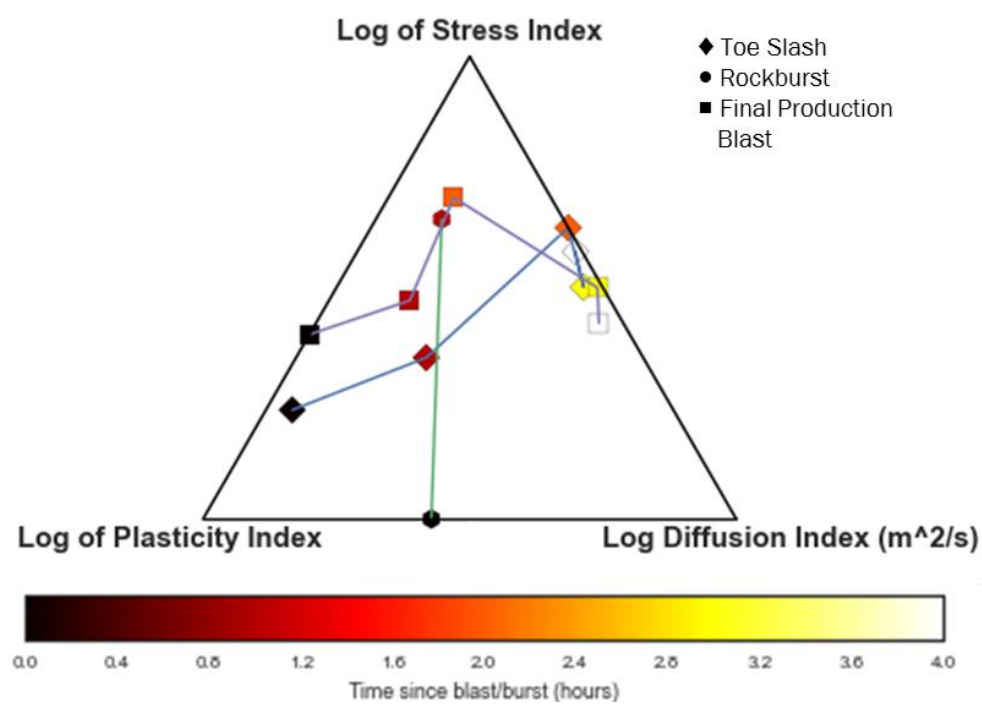

Figure 12 Ternary plot of dynamic parameterisation of the events shown in Figure 7. Events are coloured by the elapsed time, in hours, since the blast or burst. The lines show the time evolution of each day considered 
The final production blast, shown with squares in the ternary diagram in Figure 12, generates seismicity that is very similar to the toe slash. However, immediately after the blast, the rock mass experiences less deformation during the first hour as exhibited by lower PI, suggesting that the stress transfer evolves a slower stable process as seen in the higher SI values. The seismicity, interestingly, is still compact, in both space and time. During the third hour of seismicity, there is an abrupt decrease in the complexity of the stress. However, at the end of the day, the seismicity is nearly identical in its $\mathrm{PI}, \mathrm{SI}$ and $\mathrm{DI}$ to the end of the first blast sequence.

The seismicity, which occurred after the rockburst, follows a different de-stress path. Initially, the seismicity after the rockburst is very unstable, intense and compact. However, seismicity one hour later shows a marked increase in the $\mathrm{SI}$ and a decrease in the PI and DI values.

To evaluate the cumulative effect of the observed seismicity, we interpolate the dynamic parameter clusters onto a regular grid. In Figure 13, we show the SI from a cut-plane halfway through the height of the stope. Three different one-week periods are examined, each following a blast or burst.

a)

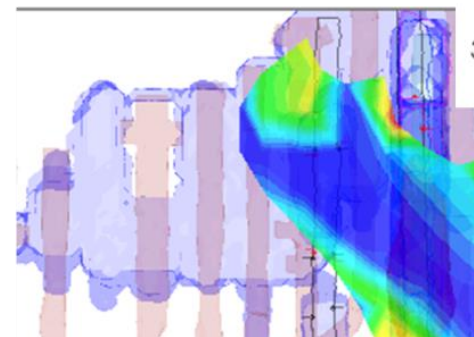

Stress Index

b)

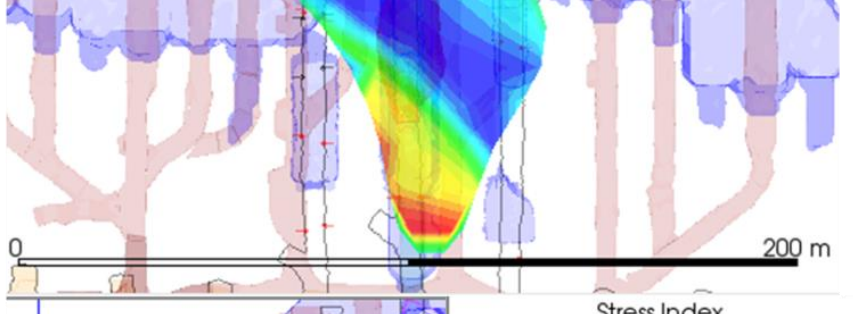

c)
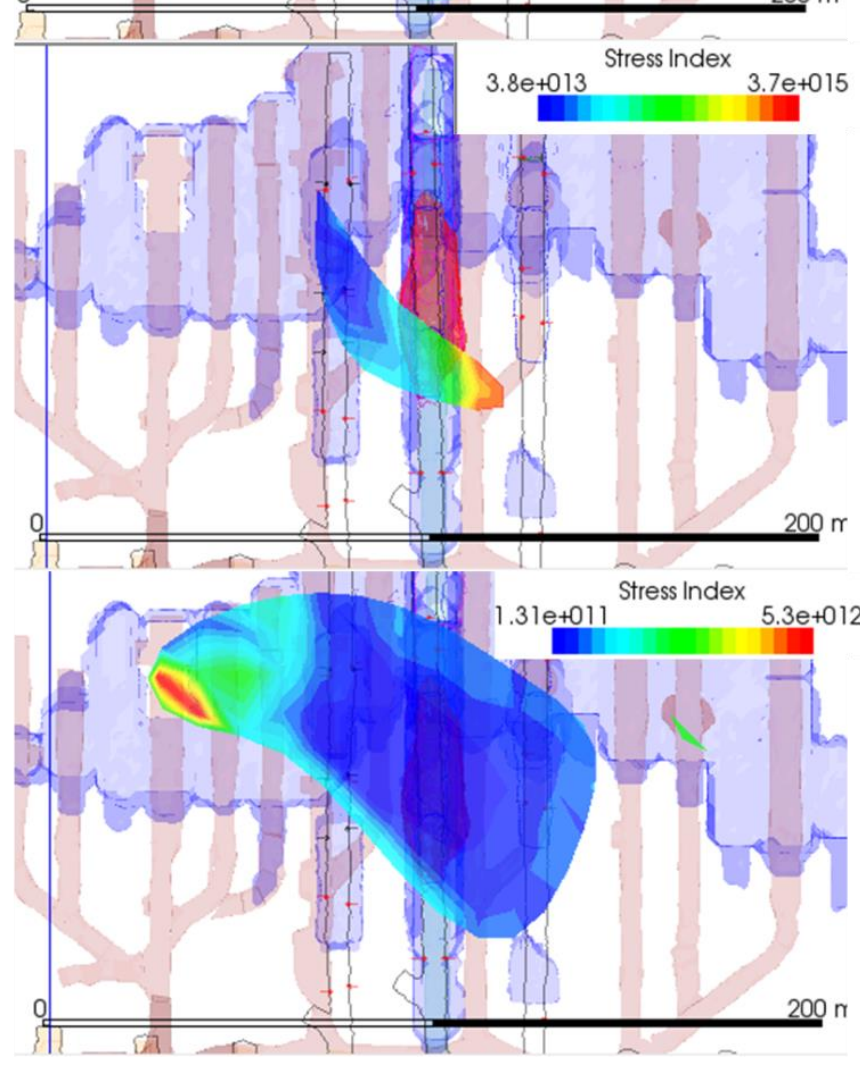

Figure 13 Contour image of the Stress Index (SI), shown halfway through the height of the stope for three different time periods, each with a different range of Stress Index values. The time periods are: a) the week following the toe slash; b) after the rockburst; and, c) after the final production blast 
After the toe slash (shown in Figure 13(a)) there is a high SI on the edges of the back-filled stopes to the north and south, outlining areas of increased stress. The seismicity following the rockburst is very spatially concentrated, with higher SI to the east, as shown in Figure 13(b). Following the final production blast, the SI decreases to quite low values - two orders of magnitude lower than the prior two time periods (Figure 13(c)). Only a small region to the west of the stope has a high $\mathrm{SI}$, with very low SI surrounding the stope. Low values of $\mathrm{SI}$ indicate that the stress has been shed from this area.

\section{Conclusion}

In this paper, we have outlined an approach for characterising variations in the size, energy budget, timing and spacing of seismicity by utilising dynamic parameters. These parameters quantify the relative variations in the complexity of the failure (Stress Index), the intensity of the deformation (Plasticity Index), and the intensity of deformation and fracturing (Diffusion Index). Using seismic moment tensors and dynamic characterisations of the collective seismic response throughout the production of a stope, we can examine changes in the deformation, and the stress and fracture state of a rock mass. By utilising these approaches we were able to identify a connection between the closure of shear-tensile cracks and regions with closely-spaced events and intense deformation.

Tracking the changes in seismicity after two blast sequences and a rockburst revealed differences in the relative contributions of changes in spatio-temporal concentration and the energy budget of the failure process. The seismicity after both the toe slash and the final production blast followed similar de-stressing paths on the ternary plot but with a different slope. For both cases, the initial seismicity involved intense, complex, compact deformation that then spreads spatially before evolving more slowly. Seismicity that occurs after a stope blast also evolves differently than seismicity that has been triggered by a rockburst, as also observed on the ternary plots.

These approaches can provide insight into the collective impact of the observed seismicity and highlight areas with unusual behaviour and allow for rapid assessment of the dynamic condition of the rock mass.

\section{Acknowledgement}

The authors would like to thank the Centre for Mining Innovation for funding this work, an anonymous hard rock mine in North America for allowing us to present the data and our colleagues at ESG Solutions who processed the data.

\section{References}

Ben-Zion, Y 2008, 'Collective behavior of earthquakes and faults: Continuum-discrete transitions, progressive evolutionary changes, and different dynamic regimes', Reviews of Geophysics, vol. 46, no. 4.

Hudson, JA, Pearce, RG \& Rogers, RM 1989, 'Source type plot for inversion of the moment tensor', Journal of Geophysical Research: Solid Earth, vol. 94, no. B1, pp. 765-774.

Hobbs, BE, Mühlhaus, HB \& Ord, A 1990, 'Instability, softening and localization of deformation', Geological Society, London, Special Publications, vol. 54, no. 1, pp. 143-165.

Kanamori, H 1983 'Magnitude scale and quantification of earthquakes', Tectonophysics, vol. 93, no. 3-4, pp. $185-199$.

Kostrov, VV 1974, 'Seismic moment and energy of earthquakes, and seismic flow of rocks', Izvestiya, Physics of the Solid Earth, no. 1, English translation, pp. 23-44.

McGarr, A 1999 'On relating apparent stress to the stress causing earthquake slip', Journal of Geophysical Research, vol. 104, pp. 3003-3011.

Mendecki, AJ 1997, 'Quantitative seismology and rock mass stability', in AJ Mendecki (ed.), Seismic Monitoring in Mines, Chapman and Hall, pp. 178-219.

Savage, JC \& Wood, MD 1971, 'The relation between apparent stress and stress drop', Bulletin of the Seismological Society of America, vol. 61, no. 5, pp. 1381-1388.

Trifu, C-I, Angus, D \& Shumila, V 2000, 'A fast evaluation of the seismic moment tensor for induced seismicity', Bulletin of the Seismological Society of America, vol. 90, no. 6, pp. 1521-1527.

Vavryčuk, V 2001, 'Inversion for parameters of tensile earthquakes', Journal of Geophysical Research Solid Earth, vol. 106, no. B8, pp. 16339-16355. 
